\title{
A NOTE ON COUNTABLY NORMED NUCLEAR SPACES
}

\author{
LASSE HOLMSTROM
}

\begin{abstract}
A modification of the Kōmura-Kōmura imbedding theorem is used to show that every countably normed nuclear space is isomorphic to a subspace of a nuclear Fréchet space with basis and a continuous norm. The space with basis can be chosen to be a quotient of $(s)$.
\end{abstract}

1. Introduction. By the famous Kōmura-Kōmura imbedding theorem [5] every nuclear Fréchet space is isomorphic to a subspace of $(s)^{\mathbf{N}}$, where $(s)$ is the space of rapidly decreasing sequences. As a corollary, every nuclear Fréchet space is isomorphic to a subspace of a nuclear Fréchet space with basis. Since $(s)^{\mathbf{N}}$ does not admit a continuous norm, we can ask to what extent this corollary holds for spaces with a continuous norm. We will show that a nuclear Fréchet space with a continuous norm is isomorphic to a subspace of a nuclear Fréchet space with basis and a continuous norm if (and only if) it is countably normed. (The concept of countably normedness was very important in constructing the first example of a nuclear Fréchet space without the bounded approximation property (see [1]).) Moreover, the space with basis can be chosen to be a quotient of $(s)$. The proof is a modification of the standard proof of the Kōmura-Kōmura theorem.

The author is grateful to Professor D. Vogt for useful discussions during his visit to Clarkson College in Fall 1981.

2. Countably normed spaces. Let $E$ be a Fréchet space which admits a continuous norm. The topology of $E$ can then be defined by an increasing sequence $\left(\|\cdot\|_{k}\right)$ of norms (the index set is $\mathbf{N}=\{1,2, \ldots\}$ ). Let $E_{k}$ denote $E$ equipped with the norm $\|\cdot\|_{k}$ only and let $\hat{E}_{k}$ be the completion of $E_{k}$. The identity mapping $E_{k+1} \rightarrow E_{k}$ has a unique extension $\phi_{k}: \hat{E}_{k+1} \rightarrow \hat{E}_{k}$ and this latter mapping is called canonical. The space $E$ is said to be countably normed if the system $\left(\|\cdot\|_{k}\right)$ can be chosen in such a way that each $\phi_{k}$ is injective.

To give an example of a countably normed space, assume that $E$ has an absolute basis i.e. there is a sequence $\left(x_{n}\right)$ in $E$ such that every $x \in E$ has a unique absolutely converging expansion $x=\Sigma_{n} \xi_{n} x_{n}$, where $\left(\xi_{n}\right)$ is a sequence of scalars. Then $E$ is isomorphic to the Köthe sequence space

$$
K(a)=K\left(a_{n}^{k}\right)=\left\{\left(\xi_{n}\right) \|\left.\left(\xi_{n}\right)\right|_{k}=\sum_{n}\left|\xi_{n}\right| a_{n}^{k}<\infty \forall k\right\},
$$

Received by the editors February 17, 1982.

1980 Mathematics Subject Classification. Primary 46A06, 46A12, 46A45.

Key words and phrases. Nuclear Fréchet space, countably normed, nuclear Köthe space. 
where $a_{n}^{k}=\left\|x_{n}\right\|_{k}$ (cf. $\left.[6,10.1]\right)$. The topology of $K(a)$ is defined by the norms $|\cdot|_{k}$. The completions $\left(K(a)_{k}\right)^{\wedge}$ can be isometrically identified with $l_{1}$ and then the canonical mapping $\phi_{k}: l_{1} \rightarrow l_{1}$ is the diagonal transformation $\left(\xi_{n}\right)_{n} \mapsto\left(\left(a_{n}^{k} / a_{n}^{k+1}\right) \xi_{n}\right)_{n}$ which is clearly injective. Therefore $E$ is countably normed.

Consider now a nuclear Fréchet space $E$ which admits a continuous norm. The topology of $E$ can be defined by a sequence $\left(\|\cdot\|_{k}\right)$ of Hilbert norms, that is, $\|x\|_{k}=\langle x, x\rangle_{k}, x \in E$, where $\langle\cdot, \cdot\rangle_{k}$ is an inner product on $E$. The following result is due to Ed Dubinsky and the proof will be contained in [3].

THEOREM 1. If a nuclear Fréchet space $E$ is countably normed, then the topology of $E$ can be defined by a sequence of Hilbert norms such that the canonical mappings $\phi_{k}$ : $\hat{E}_{k+1} \rightarrow \hat{E}_{k}$ are injective.

Suppose finally that $\left(x_{n}\right)$ is a basis of $E$. Since $\left(x_{n}\right)$ is necessarily absolute $[6,10.2 .1], E$ can be identified with a Köthe space $K(a)$. By the Grothendieck-Pietsch nuclearity criterion $[6,6.1 .2]$, for every $k$ there is $l$ with $\left(a_{n}^{k} / a_{n}^{l}\right) \in l_{1}$ : Conversely, if the matrix $\left(a_{n}^{k}\right)$ with $0<a_{n}^{k} \leqslant a_{n}^{k+1}$ satisfies this criterion, then the Köthe space $K(a)$ defined through (1) is a nuclear Fréchet space with a continuous norm and the sequence of coordinate vectors constitutes a basis. In particular, $(s)=K\left(n^{k}\right)$. The topology of such a nuclear Köthe space can also be defined by the sup-norms, $\left|\left(\xi_{n}\right)\right|_{k, \infty}=\sup _{n}\left|\xi_{n}\right| a_{n}^{k}$.

3. An imbedding theorem. We are now ready to prove the following characterization of countably normed nuclear spaces.

THEOREM 2. Let E be a nuclear Fréchet space which admits a continuous norm. Then the following two conditions are equivalent:

(i) $E$ is countably normed,

(ii) $E$ is isomorphic to a subspace of a nuclear Köthe space which admits a continuous norm.

Moreover, the Köthe space in (ii) can be chosen to be a quotient of $(s)$.

Proof. As explained in the introduction, a nuclear Köthe space with a continuous norm is countably normed. Since countably normedness is inherited by subspaces (e.g. [1, VI, 3.1.4]), the implication (ii) $\Rightarrow$ (i) is clear.

To prove (i) $\Rightarrow$ (ii) we choose a sequence $\left(\|\cdot\|_{k}\right)$ of Hilbert norms defining the topology of $E$ such that each canonical mapping $\phi_{k}: \hat{E}_{k+1} \rightarrow \hat{E}_{k}$ is injective (Theorem 1). Let $U_{k}=\left\{x \in E \mid\|x\|_{k} \leqslant 1\right\}$ and identify $\left(\hat{E}_{k}\right)^{\prime}$ with

$$
E_{k}^{\prime}=\left\{f \in E^{\prime}\left|\|f\|_{k}^{\prime}=\sup _{x \in U_{k}}\right|\langle x, f\rangle \mid<\infty\right\} .
$$

Then $\phi_{k}^{\prime}: E_{k}^{\prime} \rightarrow E_{k+1}^{\prime}$ is simply the inclusion mapping. As a Hilbert space, $E_{k+1}^{\prime}$ is reflexive. Using this and the fact that $\phi_{k}: E_{k+1} \rightarrow E_{k}$ is injective, one sees easily that $\phi_{k}^{\prime}\left(E_{k}^{\prime}\right)=E_{k}^{\prime}$ is dense in $E_{k+1}^{\prime}$.

As in the standard proof of the Kōmura-Kōmura theorem (e.g. [6, 11.1.1]) we can construct in each $E_{k}^{\prime}$ a sequence $\left(f_{n}^{(k)}\right)_{n}$ of functionals with the following properties: 


$$
U_{k}^{\circ} \subset\left\{f_{n}^{(k)} \mid n \in \mathbf{N}\right\}^{\circ \circ},
$$

$\left\{n^{l} f_{n}^{(k)} \mid n \in \mathbf{N}\right\}$ is equicontinuous for every $l$.

Now set $g_{n}^{(1)}=f_{n}^{(1)}, n \in \mathbf{N}$, and using the fact that $E_{1}^{\prime}$ is dense in every $E_{k}^{\prime}$ choose $g_{n}^{(k)} \in E_{1}^{\prime}, k \geqslant 2, n \in \mathbf{N}$, with

$$
\left\|f_{n}^{(k)}-g_{n}^{(k)}\right\|_{k}^{\prime}<2^{-n}
$$

In the construction of the desired Köthe space $K(a)$ we will use two indices $k$ and $n$ to enumerate the coordinate basis vectors. First, set

$$
a_{k n}^{l}=2^{k} n^{2 l}, \quad k, n \in \mathbf{N}, l>k .
$$

Then choose $a_{k n}^{k}, a_{k n}^{k-1}, \ldots, a_{k n}^{1}$ so that

$$
\begin{gathered}
1>a_{k n}^{k} \geqslant a_{k n}^{k-1} \geqslant \cdots \geqslant a_{k n}^{1}>0, \quad k, n \in \mathbf{N}, \\
\frac{a_{k n}^{l+1}}{a_{k n}^{l+2}} \geqslant \frac{a_{k n}^{l}}{a_{k n}^{l+1}}, \quad k, n \in \mathbf{N}, l \leqslant k, \\
a_{k n}^{l} \leqslant \frac{1}{\left\|g_{n}^{(k)}\right\|_{l}^{\prime}}, \quad k, n \in \mathbf{N}, l \leqslant k .
\end{gathered}
$$

Note that (7) holds trivially for $l>k$. Consequently, if $K\left(a_{k n}^{l}\right)=K(a)$ is nuclear, then it is also isomorphic to a quotient space of $(s)$ [2, Theorem 2.4]. But by (7), (5) and (6) for every $l \geqslant 2$,

$$
\begin{aligned}
\sum_{k=1}^{\infty} \sum_{n=1}^{\infty} \frac{a_{k n}^{l}}{a_{k n}^{l+1}} & =\sum_{k=1}^{l-1} \sum_{n=1}^{\infty} \frac{a_{k n}^{l}}{a_{k n}^{l+1}}+\sum_{k=1}^{\infty} \sum_{n=1}^{\infty} \frac{a_{k n}^{l}}{a_{k n}^{l+1}} \leqslant \sum_{k=1}^{l-1} \sum_{n=1}^{\infty} \frac{a_{k n}^{l}}{a_{k n}^{l+1}}+\sum_{k=l}^{\infty} \sum_{n=1}^{\infty} \frac{a_{k n}^{k}}{a_{k n}^{k+1}} \\
& <(l-1) \sum_{n=1}^{\infty} \frac{1}{n^{2}}+\sum_{k=1}^{\infty} \sum_{n=1}^{\infty} \frac{1}{2^{k} n^{2(k+1)}}<\infty .
\end{aligned}
$$

To imbed $E$ into $K(a)$ we set $A x=\left(\left\langle x, g_{n}^{(k)}\right\rangle\right)_{k, n}, x \in E$. We have to show that $A x \in K(a), A: E \rightarrow K(a)$ is a continuous injection and that $A^{-1}: A(E) \rightarrow E$ is also continuous.

Fix $l \geqslant 2$. Applying (3) to the sequences $\left(f_{n}^{(k)}\right)_{n}, k=1, \ldots, l-1$, we can find an index $p \geqslant l$ and a constant $C$ such that

$$
\sup _{k<l, n} 2^{k} n^{2 l}\left|\left\langle x, f_{n}^{(k)}\right\rangle\right| \leqslant C\|x\|_{p}, \quad x \in E .
$$

From (5), (8) and (4) we then get for every $x \in E$,

$$
\begin{aligned}
|A x|_{l, \infty}= & \sup _{k, n} a_{k n}^{l}\left|\left\langle x, g_{n}^{(k)}\right\rangle\right| \leqslant \sup _{k<l, n} a_{k n}^{l}\left|\left\langle x, g_{n}^{(k)}\right\rangle\right|+\sup _{k \geqslant l, n} a_{k n}^{l}\left|\left\langle x, g_{n}^{(k)}\right\rangle\right| \\
\leqslant & \sup _{k<l, n} 2^{k} n^{2 l}\left|\left\langle x, g_{n}^{(k)}\right\rangle\right|+\sup _{k \geqslant l, n} \frac{1}{\left\|g_{n}^{(k)}\right\|_{l}^{\prime}}\left|\left\langle x, g_{n}^{(k)}\right\rangle\right| \\
\leqslant & \sup _{k<l, n} 2^{k} n^{2 l}\left\|g_{n}^{(k)}-f_{n}^{(k)}\right\|_{k}^{\prime}\|x\|_{k} \\
& +\sup _{k<l, n} 2^{k} n^{2 l}\left|\left\langle x, f_{n}^{(k)}\right\rangle\right|+\|x\|_{l} \leqslant C^{\prime}\|x\|_{p},
\end{aligned}
$$

where $C^{\prime}=\sup _{n} n^{2 l} 2^{l-n}+C+1<\infty$. 
Consequently, $A x \in K(a)$ and $A: E \rightarrow K(a)$ is continuous. From (2) it follows that for every $x \in E$,

$$
\|x\|_{l}=\sup _{f \in U_{l}^{\circ}}|\langle x, f\rangle| \leqslant \sup _{n}\left|\left\langle x, f_{n}^{(l)}\right\rangle\right| \text {. }
$$

Further, since $a_{l n}^{l+1}>1$,

$$
\begin{aligned}
\sup _{n}\left|\left\langle x, f_{n}^{(l)}\right\rangle\right| & \leqslant \sup _{n}\left|\left\langle x, f_{n}^{(l)}-g_{n}^{(l)}\right\rangle\right|+\sup _{n}\left|\left\langle x, g_{n}^{(l)}\right\rangle\right| \\
& \leqslant \sup _{n}\left\|f_{n}^{(l)}-g_{n}^{(l)}\right\|_{l}^{\prime}\|x\|_{l}+\sup _{k, n} a_{k n}^{l+1}\left|\left\langle x, g_{n}^{(k)}\right\rangle\right| \\
& \leqslant \frac{1}{2}\|x\|_{l}+|A x|_{l+1, \infty} .
\end{aligned}
$$

Thus, by (9) and (10) we have for every $x \in E$,

$$
\|x\|_{l} \leqslant 2|A x|_{l+1, \infty} \text {. }
$$

Since $l$ was arbitrary, this shows that $A$ is injective and that $A^{-1}: A(E) \rightarrow E$ is continuous.

Finally we remark that is is not possible to find a single nuclear Frechet space with basis and a continuous norm containing all countably normed nuclear spaces as subspaces. In fact, it was shown in [4] that not even any countable collection of nuclear Fréchet spaces with basis and a continuous norm contains all such spaces as subspaces.

\section{REFERENCES}

1. E. Dubinsky, The structure of nuclear Fréchet spaces, Lecture Notes in Math., vol. 720, Springer-Verlag, Berlin-Heidelberg-New York, 1979.

2. E. Dubinsky and W. Robinson, Quotient spaces of ( $s$ ) with basis, Studia Math. 63 (1978), 267-281.

3. E. Dubinsky and D. Vogt, Fréchet spaces with quotients failing the bounded approximation property (to appear).

4. L. Holmström, Universal classes of nuclear Köthe spaces with a continuous norm, J. Functional Anal. 48 (1982), 12-19.

5. T. Kōmura and Y. Kōmura, Über die Einbettung der nuklearen Räume in $(s)^{A}$, Math. Ann. 162 (1966), 284-288.

6. A. Pietsch, Nuclear locally convex spaces, Springer-Verlag, Berlin-Heidelberg-New York, 1972.

Department of Mathematics and Computer Science, Clarkson College of Technology, POTSDAM, NEW YORK 13676

Current address: Department of Mathematics, University of Helsinki, Hallituskatu 15, 00100 Helsinki 10, Finland 